\title{
ROOTS OF INVERTIBLY WEIGHTED SHIFTS WITH FINITE DEFECT
}

\begin{abstract}
GERARD E. KEOUGH ${ }^{1}$
ABSTRACT. Let $T$ be a unilateral invertibly weighted shift; i.e., $T$ maps a square-summable vector sequence $\left\{x_{0}, x_{1}, \ldots\right\}$ from a Hilbert space $H$ to the sequence $\left\{0, A_{0} x_{0}, A_{1} x_{1}, \ldots\right\}$, where $\left\{A_{n}\right\}$ is a uniformly bounded sequence of invertible operators on $H$. If $S_{0}$ is the identity operator on $H$, and $S_{n}=A_{n-1} A_{n-2} \cdots A_{0}$ for $n \geqslant 1$, then $T$ is unitarily equivalent to multiplication by the variable $Z$ on the space $H^{2}(T)$ consisting of formal series $\Sigma x_{n} Z^{n}$ having coefficients $x_{n} \in H$ which satisfy $\Sigma\left\|S_{n} x_{n}\right\|^{2}<+\infty$. The commutant of this multiplication consists of formal series $\sum F_{n} Z^{n}$ which define bounded operators on $H^{2}(T)$ - where each $F_{n}$ is an operator on $H$, and the action of such a series on an element of $H^{2}(T)$ is given by the Cauchy product of the two series. Using these characterizations, it is shown that if $H$ has - finite dimension $m \geqslant 2$, then $T$ has an $n$th root only if $n$ divides $m$. Examples are given of shifts $T$ with (a) $m=2$, but $T$ has no square root, and (b) $m=4, T$ has a square root, but no fourth root.
\end{abstract}

Many theorems encountered in the study of Hilbert space operators were originally formulated and are now clearly understood only in response to the careful study of particular (classes of) operators. However, few specific classes of operators are well characterized. Perhaps the widest collection of nonnormal operators for which extensive results have been obtained are the scalar- and operator-weighted shifts. Indeed, Halmos [4] often appeals to representatives of these classes for an appropriate example or counterexample in the Hilbert space problem book.

Allen Shields [7] has collected and unified much of the theory of scalar-weighted shifts. In this paper we extend the framework developed by Shields for the scalar-weighted case to the operator-weighted case. $\$ 1$ contains a characterization of the commutant of an invertibly weighted shift. A matricial description has been obtained by Lambert [5], but here the result is obtained within the language of formal power series with operator coefficients acting as operators on a space of formal power series with vector coefficients. Then, using this characterization, $\$ 2$ obtains an existence result on the roots of an invertibly weighted shift. Specifically, if $T$ is an invertibly weighted shift for which the kernel of $T^{*}$ (the adjoint of $T$ ) has dimension $m$, where $2 \leqslant m<+\infty$, then $T$ has an $n$th root only if $n$ divides $m$.

Received by the editors March 1, 1983 and, in revised form, August 8, 1983. These results were presented to the American Mathematical Society at the 87th Summer Meeting at Albany, New York, August 1983.

1980 Mathematics Subject Classification. Primary 47B99.

Key words and phrases. Invertibly weighted shift, commutant, root of an operator.

${ }^{1}$ The author acknowledges support received during the preparation of these results from the Summer Research Grant Program of Boston College. 
Examples are given of shifts $T$ with (a) $m=2$, having no square root, and (b) $m=4$, having a square root but no fourth root.

Throughout the paper, $H$ will denote a complex Hilbert space. The term operator will mean a bounded linear transformation on a Hilbert space, and $\mathscr{B}(H)$ will denote the collection of operators on $H$. In particular, $I$ will denote the identity element of $\mathscr{H}(H) .(\cdot, \cdot)$ will generically denote the inner product on the Hilbert space in context, and $\|\cdot\|$ the induced norm. If $F \in \mathscr{B}(H)$, then $\{F\}^{\prime}$ is the commutant of $F$. Lastly, if $m$ and $n$ are positive integers, then $m \bmod n$ is the remainder upon division of $m$ by $n$.

1. A model for invertibly weighted shifts. Let $l_{2}(H)$ denote the Hilbert space of all square-summable sequences in $H . I W l_{2}(H)$ consists of all those operators $T$ on $l_{2}(H)$ whose action is given as

$$
T\left\{x_{0}, x_{1}, \ldots\right\}=\left\{0, A_{0} x_{0}, A_{1} x_{1}, \ldots\right\},
$$

where $\left\{A_{n}\right\}$ is a uniformly bounded sequence of invertible operators on $H$.

Let $T \in I W l_{2}(H)$ and suppose $\left\{A_{n}\right\}$ is the corresponding sequence of invertible operators which defines $T$. (Notation. $T \sim\left\{A_{n}\right\}$.) Let $S_{0}=I$ and $S_{n}=A_{n-1} A_{n-2}$ $\cdots A_{0}$ for $n \geqslant 1 . H^{2}(T)$ consists of all formal power series $\sum x_{n} Z^{n}$, where $\left\{x_{n}\right\}$ is a sequence of vectors from $H$ with the property that $\Sigma\left\|S_{n} x_{n}\right\|^{2}$ is finite. $H^{2}(T)$ is easily seen to be a Hilbert space with respect to the inner product

$$
\left(\sum x_{n} Z^{n}, \sum y_{n} Z^{n}\right)=\sum\left(S_{n} x_{n}, S_{n} y_{n}\right) \text {. }
$$

The operator $M_{z}$ is defined on $H^{2}(T)$ by setting $M_{z}\left(\sum x_{n} Z^{n}\right)=\sum x_{n} Z^{n+1}$. If we define a transformation $W: l_{2}(H) \rightarrow H^{2}(T)$ by setting $W\left\{x_{n}\right\}=\Sigma S_{n}^{-1} x_{n} Z^{n}$, then $W$ is a unitary transformation which satisfies $M_{z} W=W T$. Consequently, $M_{z}$ is unitarily equivalent to $T$. Thus, $M_{z}$ is indeed bounded and $\left\|M_{z}\right\|=\|T\|$.

Let $\left\{F_{n}\right\}$ be a sequence of operators on $H$, and let $\phi$ denote the formal power series $\sum F_{n} Z^{n}$. The series for $\phi$ gives rise to a mapping with domain $H^{2}(T)$, denoted $M_{\phi}$, by setting

$$
M_{\phi}\left(\sum x_{n} Z^{n}\right)=\sum y_{n} Z^{n}
$$

where for each index $n$ we define $y_{n}=\sum_{k=0}^{n} F_{k} x_{n-k}$. That is, $M_{\phi}$ is defined by formally computing the Cauchy product of the series for $\phi$ (on the left) with $\sum x_{n} Z^{n}$ (on the right). Let $H^{\infty}(T)$ denote the collection of all formal power series $\phi=\Sigma F_{n} Z^{n}$ for which $M_{\phi} H^{2}(T) \subseteq H^{2}(T)$. Since each element of $H^{\infty}(T)$ defines a closed transformation, $H^{\infty}(T)$ consists of operators on $H^{2}(T)$. Further, if $\phi, \psi \in H^{\infty}(T)$, then $M_{\phi} M_{\psi}=M_{\phi \psi}$, where $\phi \psi$ denotes the formal Cauchy product of $\phi$ (on the left) with $\psi$ (on the right). Note that $M_{z}$ is just $M_{\phi}$ when $\phi=I Z^{1}$.

THEOREM 1.1. $H^{\infty}(T)=\left\{M_{z}\right\}^{\prime}$.

Proof. Clearly, $H^{\infty}(T) \subseteq\left\{M_{z}\right\}^{\prime}$. Suppose, however, $D \in\left\{M_{z}\right\}^{\prime}$. If $x \in H$, then $D\left(x Z^{0}\right)=\sum x_{n} Z^{n} \in H^{2}(T)$, for some sequence $\left\{x_{n}\right\} \subseteq H$. For each index $n$, defined $F_{n} x=x_{n}$. That each $F_{n}$ is then well defined follows from the fact that the 
sequence $\left\{S_{n}\right\}$ is a sequence of invertible operators. To see that each $F_{n}$ is bounded, observe that

$$
\left\|S_{n} F_{n} x\right\|^{2}=\left\|S_{n} x_{n}\right\|^{2} \leqslant \sum\left\|S_{k} x_{k}\right\|^{2}=\left\|D\left(x Z^{0}\right)\right\|^{2} \leqslant\|D\|^{2} \cdot\|x\|^{2} .
$$

Thus, $\left\|F_{n}\right\| \leqslant\left\|S_{n}^{-1}\right\| \cdot\|D\|$. Lastly, if $x, y \in H$ and $j \geqslant i \geqslant 0$,

$$
\begin{aligned}
\left(D\left(x Z^{i}\right), y Z^{j}\right) & =\left(D M_{z}^{i}\left(x Z^{0}\right), y Z^{j}\right)=\left(M_{z}^{i} D\left(x Z^{0}\right), y Z^{j}\right) \\
& =\left(\sum F_{n} x Z^{n+i}, y Z^{j}\right)=\left(M_{\phi}\left(x Z^{i}\right), y Z^{j}\right)
\end{aligned}
$$

where $\phi=\Sigma F_{n} Z^{n}$. Thus, $D \in H^{\infty}(T)$.

For each index $k \geqslant 0$, let $\mathbb{Q}_{k}(T)=\left\{F \in \mathscr{B}(H) \mid \phi=F Z^{k} \in H^{\infty}(T)\right\}$. Note that if $\phi=F Z^{k} \in H^{\infty}(T)$, then $\psi=F Z^{k+1} \in H^{\infty}(T)$ as well, since $M_{\phi} M_{z}=M_{\psi}$; consequently, $\left\{\mathbb{Q}_{k}(T)\right\}$ is an increasing sequence. Further, if $F \in \mathbb{Q}_{k}(T)$ and $G \in \mathbb{Q}_{j}(T)$, then $F+G \in \mathbb{Q}_{\max \{k, j\}}(T)$, and $F G \in \mathbb{Q}_{k+j}(T)$. Thus, both $\mathcal{Q}_{0}(T)$ and $\mathcal{Q}(T)=$ $\cup_{k \geqslant 0} \mathscr{Q}_{k}(T)$ are algebras containing the identity $I$.

Proposition 1.2. Let $F \in \mathscr{B}(H)$. Then $F \in \mathbb{Q}_{k}(T)$ if, and only if, $\sup _{n}\left\|S_{n+k} F S_{n}^{-1}\right\|$ is finite.

Proof. Let $V_{k}$ denote the unilateral shift of multiplicity $k$ on $l_{2}(H)$. If $W$ denotes the unitary transformation defined previously which satisfies $M_{z} W=W T$, then it is easy to see that $W^{*} M_{\phi} W=V_{k} D$ on $l_{2}(H)$, where $\phi=F Z^{k}$, and $D$ is the diagonal operator on $l_{2}(H)$ whose action is defined as $\left\{x_{n}\right\} \rightarrow\left\{S_{n+k} F S_{n}^{-1} x_{n}\right\}$.

In closing this section, we remark that a special case of Theorem 1.1, where $T$ is a pure quasinormal operator and $T^{*} T$ is invertible, has appeared in a paper of Conway and Wu [1]. Also, the algebras $\left\{Q_{k}(T)\right\}$ were previously studied by A. L. Lambert and T. R. Turner [6], in determining which invertibly weighted shifts have the double commutant property. These algebras were also studied in special cases in [2] and [3].

2. Roots of shifts. Given operators $R$ and $T$ and a positive integer $n$, we say that $R$ is an $n$th root of $T$ if $R^{n}=T$. Throughout this section we assume $n$ is a fixed positive integer, $T \in I W l_{2}(H), T \sim\left\{A_{k}\right\}$, and the dimension of $H$ is $m$ for a finite integer $m \geqslant 2$.

THEOREM 2.1. Let $R$ be an nth root of T. Then $n \leqslant m$ and $m \bmod n$ is zero.

Proof. Since any roots of $T$ necessarily lie in $\{T\}^{\prime}$, we consider $R$ to be an $n$th root of $M_{z}$ on $H^{2}(T)$. Thus, suppose $\phi=\Sigma F_{k} Z^{k} \in H^{\infty}(T)$ and $\left(M_{\phi}\right)^{n}=M_{z}$. Since elements of $H^{\infty}(T)$ have unique series expansions (reason: $\left\{S_{k}\right\}$ is a sequence of invertible operators), this implies that

(a) $F_{0}^{n}=0$

(b) $\sum_{k=0}^{n-1} F_{0}^{n-1-k} F_{1} F_{0}^{k}=I$.

The minimal polynomial for $F_{0}$ is then of the form $X^{j}$ with $2 \leqslant j \leqslant m$.

First, suppose $n>m$. Then (b) can be rewritten as

$$
I=\sum_{k=0}^{j-1} F_{0}^{n-1-k} F_{1} F_{0}^{k}=F_{0}^{n-j} \cdot \sum_{k=0}^{j-1} F_{0}^{j-1-k} F_{1} F_{0}^{k} .
$$


However, $n-j>0$ and, hence, $m=\operatorname{rank}(I) \leqslant \operatorname{rank}\left(F_{0}\right)<m$, which is absurd. Therefore, $n \leqslant m$.

Next, we claim that the minimal polynomial for $F_{0}$ is exactly $X^{n}$. For suppose $F_{0}^{n-1}=0$. Once again, rewriting (b) shows

$$
I=\sum_{k=0}^{n-1} F_{0}^{n-1-k} F_{1} F_{0}^{k}=F_{0} \cdot \sum_{k=0}^{n-2} F_{0}^{n-2-k} F_{1} F_{0}^{k}
$$

which again would give $m=\operatorname{rank}(I) \leqslant \operatorname{rank}\left(F_{0}\right)<m$.

Lastly, because the minimal polynomial for $F_{0}$ is $X^{n}$, its Jordan canonical form will consist of a number of blocks of the form

$$
\left(\begin{array}{lllllll}
0 & 1 & 0 & . & . & . & 0 \\
0 & 0 & 1 & . & . & . & 0 \\
0 & 0 & 0 & 1 & . & . & 0 \\
. & . & . & . & . & . & . \\
. & . & . & . & . & 0 & 1 \\
. & . & . & . & . & . & 0
\end{array}\right),
$$

where each block contains l's above the main diagonal, 0 's elsewhere, and less than or equal to $n$ rows. The maximum rank of each block is thus $n-1$. Let $r$ be $(m \bmod n)$ and suppose $r \neq 0$. Suppose $q$ is chosen so that $m=q n+r$. By inspection, then, it is clear that $F_{0}$ has maximum rank if and only if each block in its Jordan canonical form is as large as possible. Thus, the rank of $F_{0}$ will be less than or equal to $(n-1) q+(r-1)$, since there will be at most $q$ blocks of $n$ rows and a block of $r$ rows. Again rewriting (b), we see that

$$
I=F_{0} \cdot \sum_{k=0}^{n-2} F_{0}^{n-2-k} F_{1} F_{0}^{k}+F_{1} F_{0}^{n-1} .
$$

The first summand has rank at most $(n-1) q+(r-1)$; the second at most $q$, since the rank of $F_{0}^{n-1}$ is at most $q$. That is,

$$
m=\operatorname{rank}(I) \leqslant n q-q+r-1+q=m-1 .
$$

This completes the proof.

We close the section with examples to show that while the condition $(m \bmod n)=0$ is necessary for the existence of an $n$th root of $T$, it is not sufficient. Indeed, the existence of particular roots depends largely on the structure of the sequence $\left\{\mathbb{Q}_{k}(T)\right\}$ and on the algebra $\mathbb{Q}_{0}(T)$ in particular.

EXAMPLE 2.2. Let $H$ have dimension 2 and, for each index $n \geqslant 0$, let $A_{n}$ be the operator on $H$ defined by the matrix $\left(\begin{array}{l}1 \\ 0 \\ 0\end{array}\right)$. Let $T \sim\left\{A_{n}\right\} \in I W l_{2}(H)$. For each $n \geqslant 0, S_{n}$ will be defined by the matrix $\left(\begin{array}{ll}1 & n \\ 0 & 1\end{array}\right)$. Using Proposition 1.2, one can directly compute that each $\mathbb{Q}_{k}(T)$ is exactly the algebra of operators on $H$ whose matrices have the form $\left(\begin{array}{c}a b \\ 0\end{array}\right)$, for constants $a$ and $b$, for each $k \geqslant 0$. If $M_{z}$ has a square root in $H^{\infty}(T)$, then there would exist operators $F_{0} \in \mathbb{Q}_{0}(T)$ and $F_{1} \in \mathbb{Q}_{1}(T)$ which satisfy the operator equations $F_{0}^{2}=0$ and $F_{0} F_{1}+F_{1} F_{0}=I$. The first implies that $F_{0}$ must have a matrix of the form $\left(\begin{array}{l}0 b \\ 0\end{array}\right)$, for some constant $b$. However, this will imply that $F_{0} F_{1}+F_{1} F_{0}$ has rank at most one. 
A reasonable question at this point would be: if $T \in I W l_{2}(H)$ has any roots, will it have all possible roots? The answer is no.

EXAMPLE 2.3. Let $T$ be the operator which is the square of the operator in Example 2.2. Clearly $T$ has a square root; however, it has no fourth root. To see this, note that $T$ will be defined by the sequence $\left\{A_{n}\right\}$, where for $n \geqslant 0, A_{n}$ will be represented by the matrix

$$
\left(\begin{array}{llll}
1 & 2 & 0 & 0 \\
0 & 1 & 0 & 0 \\
0 & 0 & 1 & 2 \\
0 & 0 & 0 & 1
\end{array}\right)
$$

Consequently, $S_{n}$ has matrix

$$
\left(\begin{array}{cccc}
1 & 2 n & 0 & 0 \\
0 & 1 & 0 & 0 \\
0 & 0 & 1 & 2 n \\
0 & 0 & 0 & 1
\end{array}\right)
$$

for each $n \geqslant 0$. For each $k \geqslant 0, \mathbb{Q}_{k}(T)$ can be computed to be exactly the collection of operators on $H$ which have a matrix representation of the form

$$
\left(\begin{array}{llll}
a & b & c & d \\
0 & a & 0 & c \\
e & f & g & h \\
0 & e & 0 & g
\end{array}\right)
$$

for constants $a, \ldots, h$. If $M_{z}$ has a fourth root in $H^{\infty}(T)$, there would exist operators $F_{0} \in \mathbb{Q}_{0}(T)$ and $F_{1} \in \mathbb{Q}_{1}(T)$ which satisfy equations (a) and (b) of the proof of Theorem 2.1. Let $a, \ldots, h$ be constants for which $F_{0}$ has matrix

$$
\left(\begin{array}{llll}
a & b & c & d \\
0 & a & 0 & c \\
e & f & g & h \\
0 & e & 0 & g
\end{array}\right)
$$

Note that $F_{0}$ has determinant $(a g-c e)^{2}=0$. If $a$ were zero, then one of $c$ or $e$ would be zero, and since $F_{0}^{4}=0$, this would imply $g$ is zero as well. It is then easy to argue that equation (b) of the proof will not hold for any $F_{1}$ - say by examining the upper left entry in the matrix expansion of the equation. Consequently, $a$ is nonzero, so we may as well consider $F_{0}$ to have the form

$$
\left(\begin{array}{cccc}
1 & b & c & d \\
0 & 1 & 0 & c \\
e & f & c e & h \\
0 & e & 0 & c e
\end{array}\right)
$$

In this case the upper left entry of $F_{0}^{4}$ will be $(1+c e)^{3}$, so $F_{0}$ actually has the form

$$
\left(\begin{array}{cccc}
1 & b & c & d \\
0 & 1 & 0 & c \\
-1 / c & f & -1 & h \\
0 & -1 / c & 0 & -1
\end{array}\right) .
$$


In this case we again see that equation (b) cannot be satisfied, by examining the upper left entry in its matrix expansion.

As a final note, an "algebraic $m$ th root" always exists:

EXAMPLE 2.4. Let $U_{+}$denote the forward shift on $H$; that is, let $U_{+}$be given by the matrix

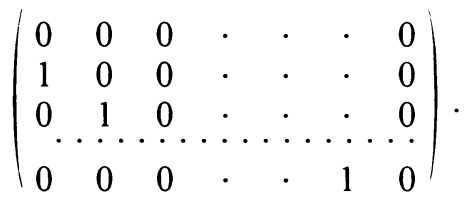

Let $U_{-}$denote the backward shift; i.e., $U_{-}$is the adjoint of $U_{+}$. Let $F_{0}=U_{-}$, and $F_{1}=\left(U_{+}\right)^{m-1}$. If $\phi$ is formally defined as the series $\phi=F_{0} Z^{0}+F_{1} Z^{1}$, then for each $k$ with $2 \leqslant k \leqslant m, \phi^{k}$ will formally have the series

$$
\left(F_{0}\right)^{k} Z^{0}+\left(\sum_{j=0}^{k-1} F_{0}^{k-1-j} F_{1} F_{0}^{j}\right) Z^{1} .
$$

Thus, $\phi^{m}$ is the series $I Z^{1}$. Hence, if $T \in I W l_{2}(H)$, and if $F_{0} \in \mathbb{Q}_{0}(T)$ and $F_{1} \in$ $Q_{1}(T), T$ will have an $m$ th root. Consequently, a sufficient condition that $T$ have an $m$ th root is available; a somewhat stronger condition, of course, would be to require that $\mathbb{Q}_{0}(T)=\mathscr{B}(H)$, or that $\sup _{n}\left\|S_{n} F S_{n}^{-1}\right\|$ be finite for all $F \in \mathscr{B}(H)$.

\section{REFERENCES}

1. John B. Conway and Pei Yuan Wu, The structure of quasinormal operators and the double commutant property, Trans. Amer. Math. Soc. 270 (1982), 641-657.

2. James A. Deddens, Another description of nest algebras, Hilbert Space Operators, Lecture Notes in Math., vol. 693, Springer-Verlag, Berlin and New York, pp. 77-85.

3. James A. Deddens and Tin Kin Wong, The commutant of analytic Toeplitz operators, Trans. Amer. Math. Soc. 184 (1973), 261-273.

4. P. R. Halmos, A Hilbert space problem book, Van Nostrand, Princeton, N. J., 1967.

5. Alan Lambert, Unitary equivalence and reducibility of invertibly weighted shifts, Bull. Austral. Math. Soc. 5 (1971), 157-173.

6. A. L. Lambert and T. R. Turner, The double commutant of invertibly weighted shifts, Duke Math. J. 39 (1972), 385-389.

7. Allen L. Shields, Weighted shift operators and analytic function theory, Math. Surveys, no. 13 (C. Pearcy, ed.), Amer. Math. Soc., Providence, R. I., 1974.

Department of Mathematics, Boston College, Chestnut Hill, Massachusetts 02167 